International Journal on Cybernetics \& Informatics (IJCI) Vol. 5, No. 4, August 2016

\title{
DESIGN AND FABRICATION OF S-BAND MIC POWER AMPLIFIER
}

\author{
NNeeraja $^{1}$, P V K Chaitanya ${ }^{2}$ and Dr. K C B Rao ${ }^{3}$ \\ ${ }^{1}$ PG Scholar, Dept. of E C E, G V P College of Engineering for Women, Visakhapatnam \\ ${ }^{2}$ Assistant Professor, Dept. of ECE, GVP College of Engineering for Women, \\ Visakhapatnam \\ ${ }^{3}$ Associate Professor, Dept. of E C E, University College of Engineering, Vizianagaram
}

\begin{abstract}
In this paper, we demonstrate an approach to design FET (pHEMT) based amplifier. The FET is from Berex Inc.The design is carried out using the measured S-parameter data of the FET.ADS is used as design tool for the design. A single-stage power amplifier demonstrated 13dB output gain from $3 G H z-4 G H z . T h e$ saturated output power of $1 W$ and the power added efficiency (PAE) up to 43\%.The amplifier is fabricated on a selective device GaAs power pHEMT process in MIC (Microwave Integrated Circuit) Technology. MICs are realized using one or more different forms of transmission lines, all characterized by their ability to be printed on a dielectric substrate.Active and passive components such as transistors/FET, thin or thick film chip capacitors and resistors are attached.
\end{abstract}

\section{KEYWORDS}

pHEMT, MIC, Power Amplifier

\section{INTRODUCTION}

Power amplifiers (PAs) are the most energy-consuming component in wireless transceivers. PAs represent more than $60 \%$ of consumed energy in the overall communication system. The trends show that the next generations of wireless communication system will require broadband, High Power Added Efficiency PA allowing reliability capabilities.

Microwave Integrated Circuit (MIC) technologies have made a great advance in various aspects. No area of the field has seen greater changes than that of microwave amplifiers and oscillators. The active devices used in amplifiers and oscillators have evolved from vacuum tubes (klystrons and magnetrons) to two terminal solid state devices (Gunn, IMPATT, Tunnel and Varactor diodes) and finally to three terminal solid state devices (GaAs FETs, and silicon bipolar transistors). At the same time, the transmission line media has changed from waveguides, to coaxial cable and finally to micro strip lines. GaAs devices address high power and high

DOI: $10.5121 /$ ijci.2016.54067 
efficiency performances. The main future challenge is to design PA with high power characteristics associated with the highest bandwidth and the smallest size as possible.

S-parameters are important in microwave design because they are easier to measure and work with at high frequencies than other kinds of parameters. They are conceptually simple, analytically convenient, and capable of providing a great insight into a measurement or design problem.A network analyzer is an instrument that measures the network parameters of electrical networks. Today, network analyzers commonly measure S- parameters because reflection and transmission of electrical networks are easy to measure at high frequencies.

\section{PHEMT DEVICE}

The characteristics of our pHEMT devices make them ideal choices for usage as high efficiency, linear power amplifiers with variable output capabilities. The high gain at low drain currents and the sham pinch off $I$ large-drain voltages enable the devices to operate very efficiently over very large portion of their I-V characteristics. Our amplifiers maintain their linearity,bandwidth and high efficiency when their drainvoltages are reduced to as low a value as $2 \mathrm{~V}$. This is accomplished without retuning the amplifiers at eachdrain voltage - the amplifiers are fixed tuned. Amplifiers with comparable performance have not beenpreviously demonstrated with any solid state devices.

The pHEMT device used is BCP120T. BCP120T is a GaAs Power pHEMT with a nominal 0.25 micron gate length and 1200 micron gate width making the product ideally suited for amplifier applications where high-gain and medium power from DC to $26 \mathrm{GHz}$. The product may be used in either wideband or narrow-band applications. The BCP120T is produced using state of the art metallization with SI3N4 passivation and is screened to assure reliability.
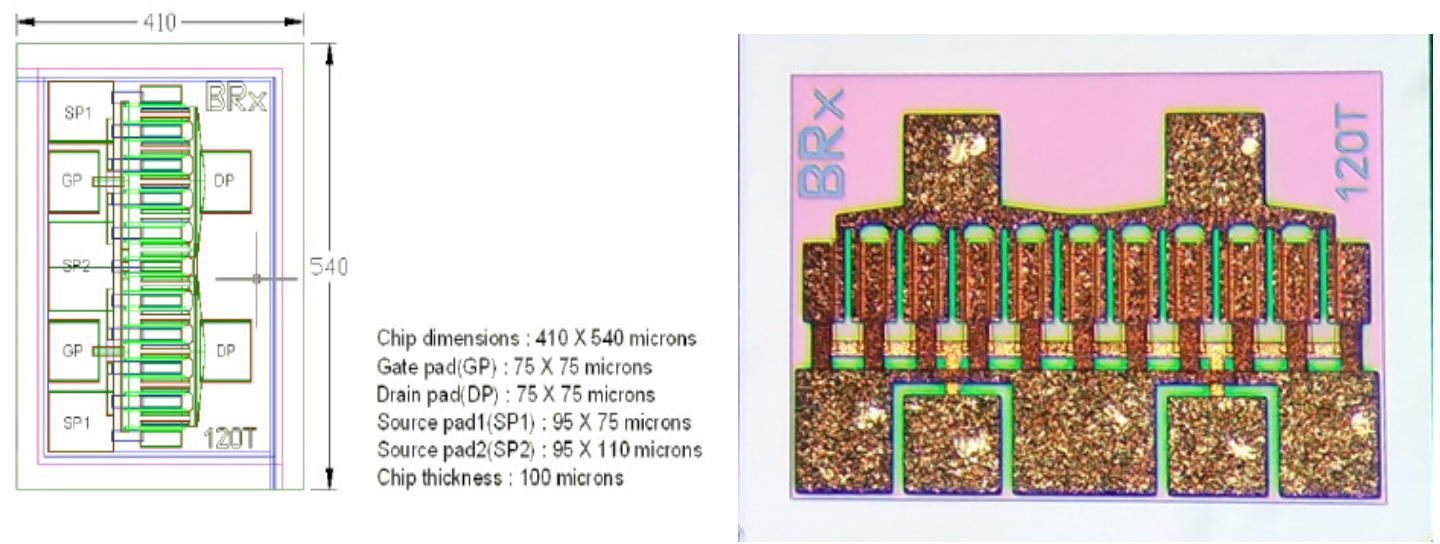

Fig1. pHEMT

At $25 \mathrm{C}$, The continuous values can be as Drain-Source Voltage Vds $8 \mathrm{~V}$, Gate-Source Voltage Vgs -3 V ,Drain Current Ids =Idss ,Forward Gate Current Igsf 10 mA ,Input Power Pin @ 3dB compression. The electrical characteristics for calculating the output power the specifications 
given as Output Power P1dB is $31.0 \mathrm{dBm} @ \mathrm{P} 1 \mathrm{~dB}(\mathrm{Vds}=8 \mathrm{~V}$, Ids $=50 \%$ Idss), Gain is $11.0 \mathrm{dBm}$ @ $\mathrm{P} 1 \mathrm{~dB}(\mathrm{Vds}=8 \mathrm{~V}$, Ids =50\% Idss) PAE is 40\% @ P1dB (Vds $=8 \mathrm{~V}$, Ids =50\% Idss).

\section{AMPLIFIER DESIGN}

The design of any practical amplifier is a multistep process. The design procedure leading to the simulation of an ideal circuit is Define a specification, Choose a topology, Choose an active device, Stabilize the device, Choose a bias circuit, Address power or noise matching conditions, Perform computer simulations and optimizations to realize the specifications. Tool used: ADS Tool is used to design the power amplifier circuit design. Advanced Design System (ADS) is an electronic design automation software system. It provides an integrated design environment to designers of RF electronic products such as mobile phones, pagers, wireless networks, satellite communications, radar systems, and high-speed data links. This chapter describes the windows, menus and basic operations or working in the ADS Design Environment performs the operations: Creating projects to organize and save your designs. Creating system diagrams, circuit schematics and EM structures. Placing circuit elements into schematics. Placing system blocks into system diagrams. Incorporating and tuning sub circuits into system diagrams and schematics. Running simulations for schematics and system diagrams, displaying output graphs, creating layouts. Sparameters for two port networks, at microwave frequencies, the parameters used for measurement have inputs and outputs expressed in power. These microwave parameters are called S-parameters. S-parameters are transmission and reflection coefficients between the incident and reflected waves. The transmission coefficients are commonly called gains or attenuation, and the reflection coefficients are directly related to VSWRs and impedances. Each parameter is typically characterized by magnitude, decibel and phase. The expression in decibel is $20 \log S_{\mathrm{ij}}$ because $\mathrm{S}$-parameters are voltage ratios of the waves.

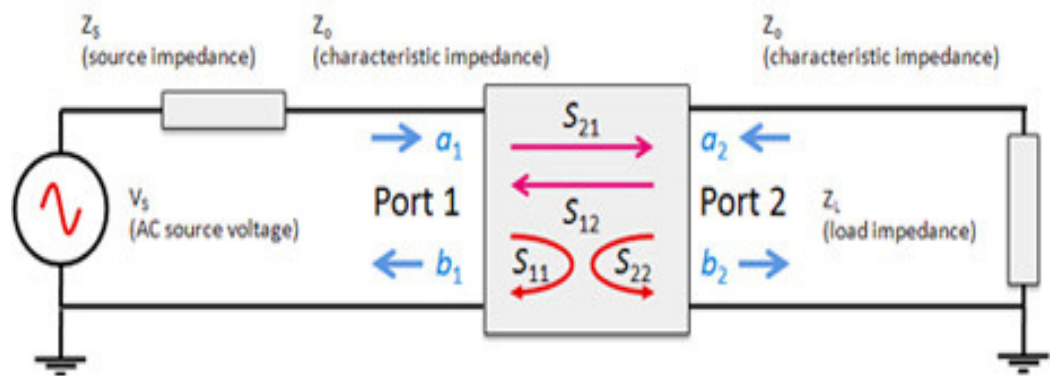

Fig 2.Block diagram of S-Parameters 
International Journal on Cybernetics \& Informatics (IJCI) Vol. 5, No. 4, August 2016

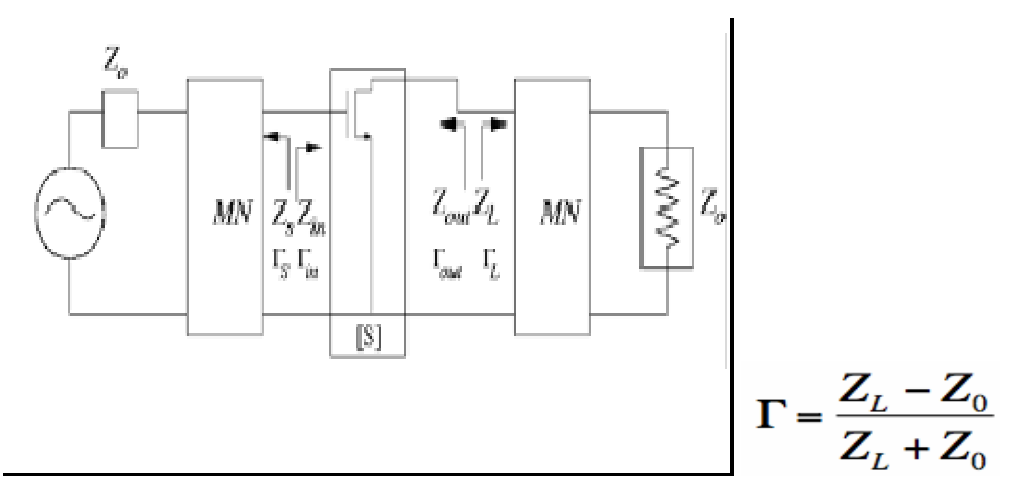

Fig 3.Matching Network

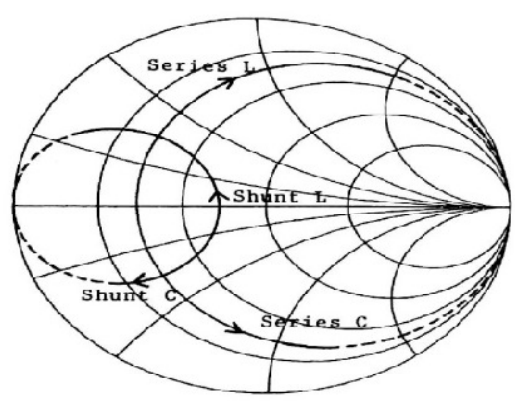

Fig 4.Smith chart

Impedance Matching Using Smith Chart: Matching a transmission line simply means terminating the line in its characteristic impedance so that the standing wave ratio of unity transmits a given power without any reflection. Also, transmission efficiency is optimum where there is no reflected power. Matching can be tried first on the load side to flatten the line, then adjustment may be made on the source side to provide maximum power transfer.

\section{Circuit Design}

Input and output matching networks are released by necessary theoretical calculations.Transmission lines are used to design the circuit.Signals at high frequencies are usually sinusoidal or can be analyzed as though they are composed of sinusoidal waves that are propagating through a component from input to output. Moreover, any signal waveform can be represented in terms of sinusoidal components using Fourier analysis. Considering all signals to be sinusoidal makes the solution of partial differential equations (3.1) and (3.2) in time domain much easier. Hence, it is appropriate to analyze transmission line equations in steady-state sinusoidal form. Both the voltage and current will be in phasor form and will vary in time and distance. Let V and I be the voltage and current phasors, respectively, at any arbitrary point on the transmission line. 
The derivatives of the voltage and current phasors from the above equations can be expressed as:

$$
\begin{aligned}
& -\frac{d V}{d z}=R I+L \frac{d I}{d t} \\
& -\frac{d I}{d z}=G V+C \frac{d V}{d t}
\end{aligned}
$$

A generalized solution of the coupled differential equations (1) and (2) can be given by,

$$
\begin{aligned}
& V(z, t)=\left(\mathrm{V}^{+} \mathrm{e}^{-\gamma z}+\mathrm{V}^{-} \mathrm{e}^{+\gamma z}\right) \mathrm{e}^{\mathrm{j} \omega t} \\
& I(z, t)=\left(I^{+} \mathrm{e}^{-\gamma z}+I^{-} \mathrm{e}^{+\gamma z}\right) \mathrm{e}^{\mathrm{j} \omega t}
\end{aligned}
$$

where $\gamma=\alpha+j \beta$ is the propagation constant

$\mathrm{V}^{+}, \mathrm{I}^{+}=$complex voltage and current amplitudes in the positive $\mathrm{z}$ direction

$\mathrm{V}^{-}, \mathrm{I}^{-}=$complex voltage and current amplitudes in the negative $\mathrm{z}$ direction

$\alpha=$ attenuation constant per unit length of the line

$\beta=$ phase constant per unit length of the line

Substituting equations (3) and (4) into (1) and (2) gives

$$
\begin{aligned}
& \frac{d V}{d z}=-(R+j \omega L) I=-Z . I \\
& \frac{d I}{d z}=-(G+j \omega C) V=-Y . V
\end{aligned}
$$

where $\mathrm{Z}=$ impedance phasor per unit length of the transmission line

$\mathrm{Y}=$ susceptance phasor per unit length of the transmission line

Transmission line parameters are considered for the design as Propagation constant $(\gamma)$, Phase velocity $\left(\mathrm{v}_{\mathrm{p}}\right)$, Characteristic impedance $\left(\mathrm{Z}_{0}\right)$, Reflection coefficient $(\Gamma)$, Voltage standing wave ratio(VSWR),

$$
\begin{gathered}
\gamma=\alpha+j \beta=[(R+j \omega L)(G+j \omega C)]^{1 / 2} \\
\mathrm{~V}_{\mathrm{p}}=\omega / \beta \\
Z 0=(Z / Y)^{1 / 2}=[(R+j \omega L) /(G+j \omega C)]^{1 / 2} \\
\Gamma=\mathrm{E}_{\mathrm{r}} / \mathrm{E}_{\mathrm{i}}=\left(\mathrm{V}-\mathrm{e}^{\gamma l} / \mathrm{V}+\mathrm{e}^{-\gamma l}\right) \\
\mathrm{VSWR}=\left|\mathrm{V}_{\text {max }} / \mathrm{V}_{\text {min }}\right|=(1+\Gamma) /(1-\Gamma)
\end{gathered}
$$


International Journal on Cybernetics \& Informatics (IJCI) Vol. 5, No. 4, August 2016

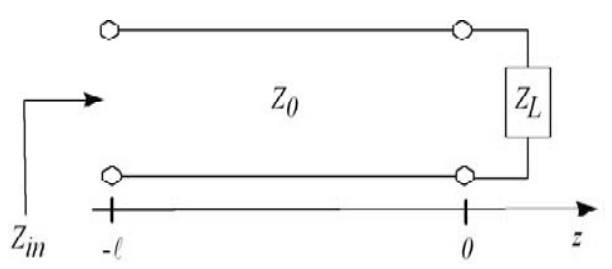

Fig 5. Transmission line terminated load impedance

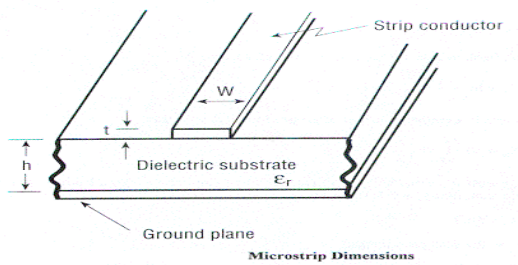

Fig 6. Microstrip

Micro strip parameters considered $\operatorname{arev}_{\mathrm{p}}=\mathrm{c} /\left(\varepsilon_{\mathrm{eff}}\right)^{1 / 2}$

$$
\begin{gathered}
Z_{\mathrm{o}}=\frac{60}{\sqrt{\varepsilon_{\text {eff }}}} \ln \left(8 \frac{\mathrm{h}}{\mathrm{W}}+\frac{\mathrm{W}}{4 \mathrm{~h}}\right) \\
\text { where } \varepsilon_{\text {eff }}=\frac{\varepsilon_{\mathrm{r}}+1}{2}+\frac{\varepsilon_{\mathrm{r}}-1}{2}\left[\left(1+12 \frac{\mathrm{h}}{\mathrm{W}}\right)^{-1 / 2}+0.04\left(1-\frac{\mathrm{W}}{\mathrm{h}}\right)^{2}\right] \text { for } \frac{\mathrm{W}}{\mathrm{h}} \leq 1 \\
Z_{\mathrm{o}}=\frac{120 \pi}{\sqrt{\varepsilon_{\text {eff }}\left[\frac{\mathrm{W}}{\mathrm{h}}+1.393+0.667 \ln \left(\frac{\mathrm{W}}{\mathrm{h}}+1.444\right)\right]}} \\
\text { where } \varepsilon_{\text {eff }}=\frac{\varepsilon_{\mathrm{r}}+1}{2}+\frac{\varepsilon_{\mathrm{r}}-1}{2}\left(1+12 \frac{\mathrm{h}}{\mathrm{W}}\right)^{-1 / 2} \text { for } \frac{\mathrm{W}}{\mathrm{h}} \geq 1
\end{gathered}
$$

Stability considerations are one of the important aspects for the design of the circuit. So ,In terms of $\mathrm{k}$ factor, the stability criteria are, unconditional stability, $\mathrm{k}>1$.0.conditional stability, $\mathrm{k}<1.0$

$$
k=\frac{\left(1-|\mathrm{S} 11|^{2}-|\mathrm{S} 22|^{2}+|\mathrm{D}|^{2}\right)}{2|\mathrm{~S} 12||\mathrm{S} 21|}
$$

where $S_{11}, S_{22}, S_{12}, S_{21}$ are the complex $S$-parameters and $D=S_{11} S_{22}-S_{12} S_{21}$. 
International Journal on Cybernetics \& Informatics (IJCI) Vol. 5, No. 4, August 2016

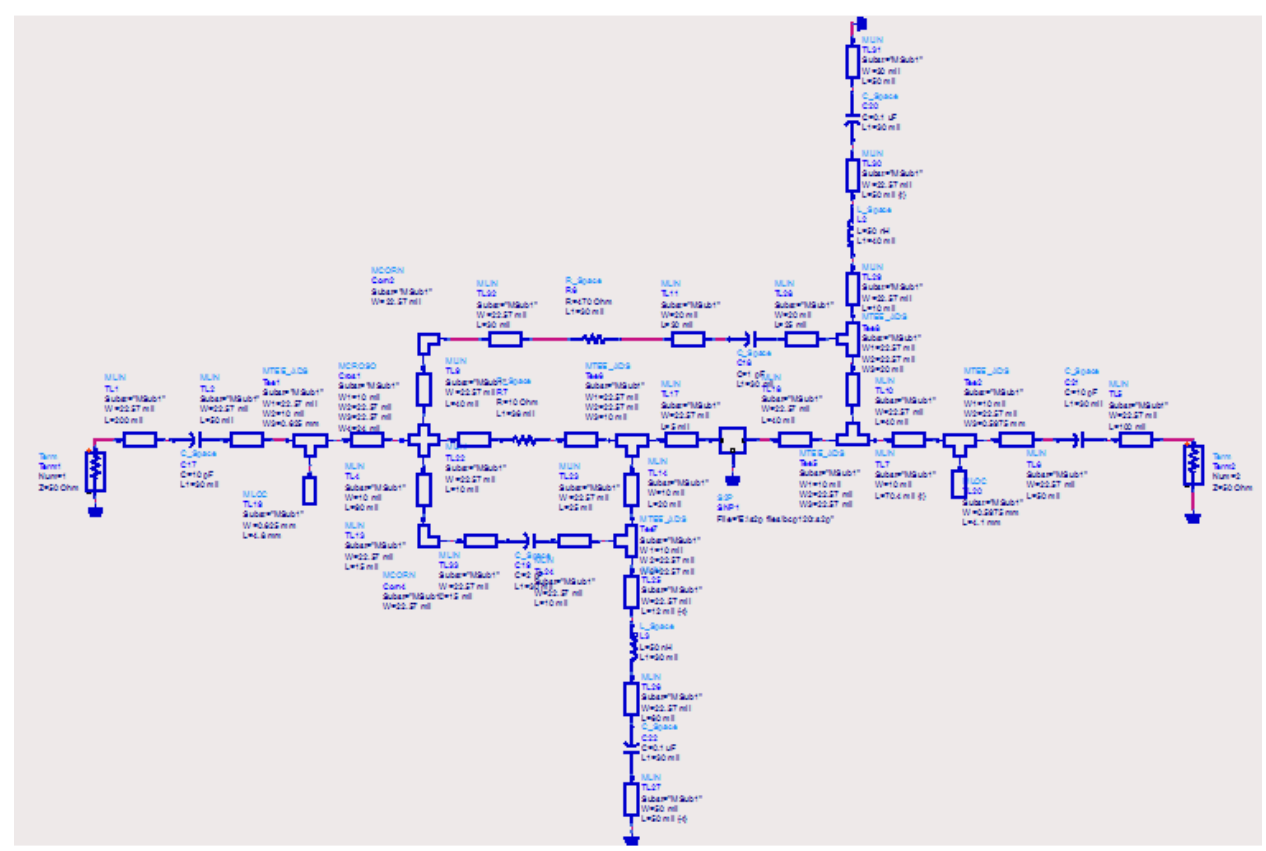

Fig 7. Schematic of power amplifier

Fig 7 is the schematic diagram of power amplifier attained from the ADS tool software after designing the circuit by considering the input and output matching network.Fig 8 is the layout resulted from the fig 7 .

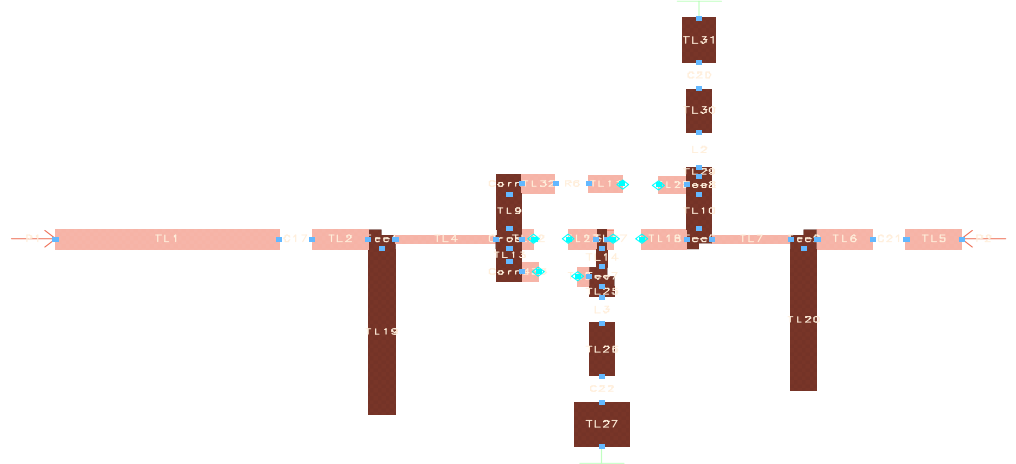

Fig 8. Layout of power amplifier

\section{FABRICATION:}

On the RT duroid material the blue print of the circuit distributed components is done and given for the circuit assembly. Assembly deals with placing the component on the fabricated circuit. 
The components can be attached by using epoxy /eutectic bonding, soldering. Any Interconnection between the transistor chip and the components can be provided by wire bonds. The IC fabrication flow using the transistor chip and lumped elements such as a chip capacitor and coil inductor.

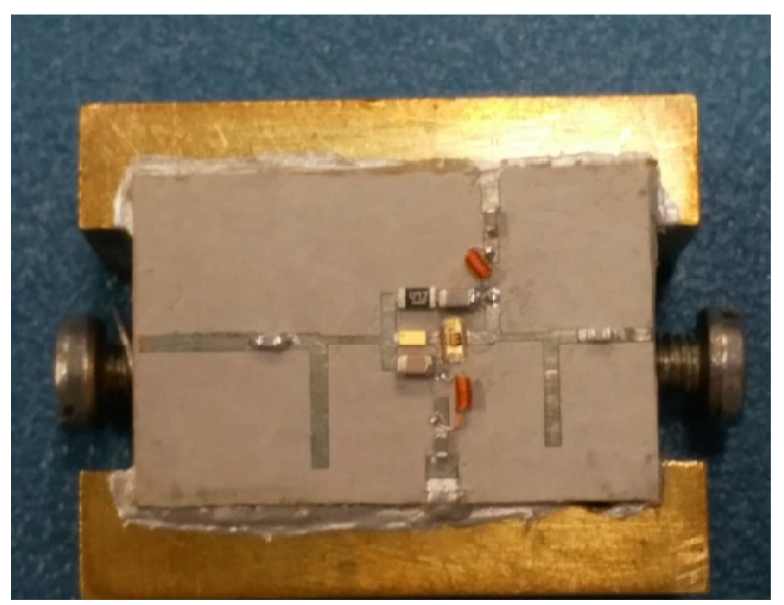

Fig 9. MIC fabricated power amplifier

\section{Amplifier Performance}

After testing the fabricated device in the network analyzer,the final transmission and reflection coefficients results are given below.The return losses are above $-18 \mathrm{~dB}$. The output power resulted is $30 \mathrm{dBm}$.

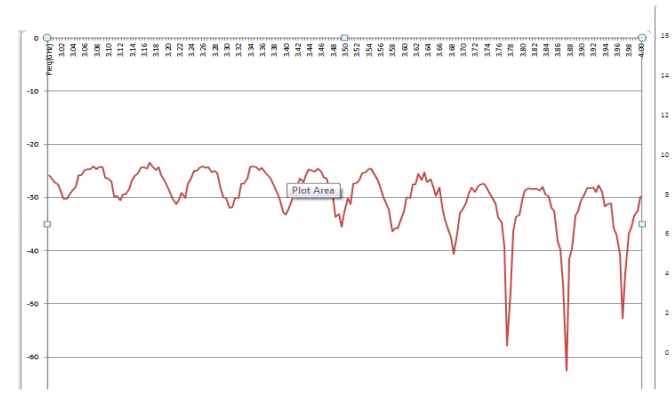

Fig 10. s22

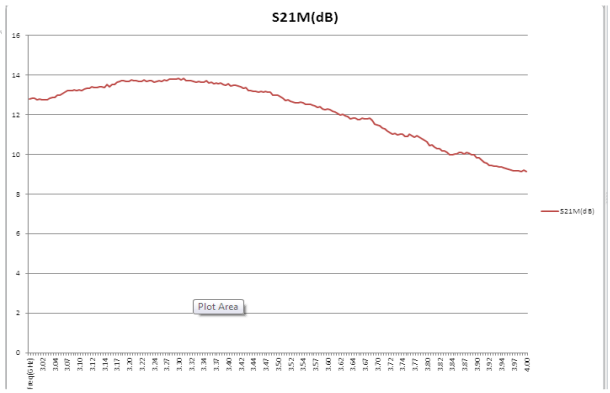

Fig 11. s21 
International Journal on Cybernetics \& Informatics (IJCI) Vol. 5, No. 4, August 2016

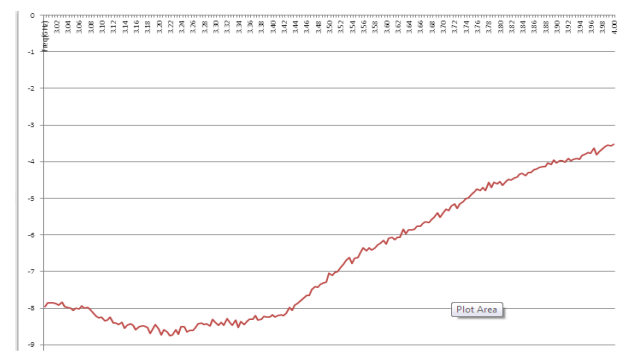

Fig 12. s11

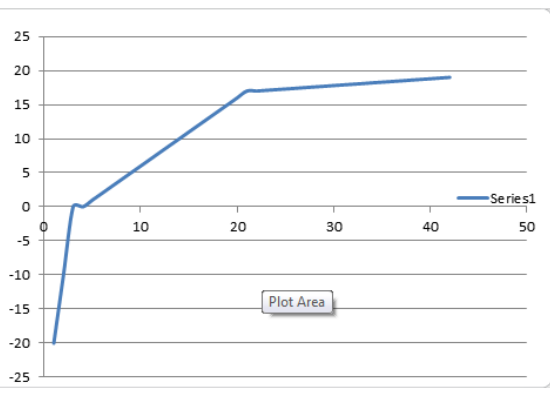

Fig 13. Output power

The final simulation results attained for the circuit shown in fig 7 are given in Fig 14.
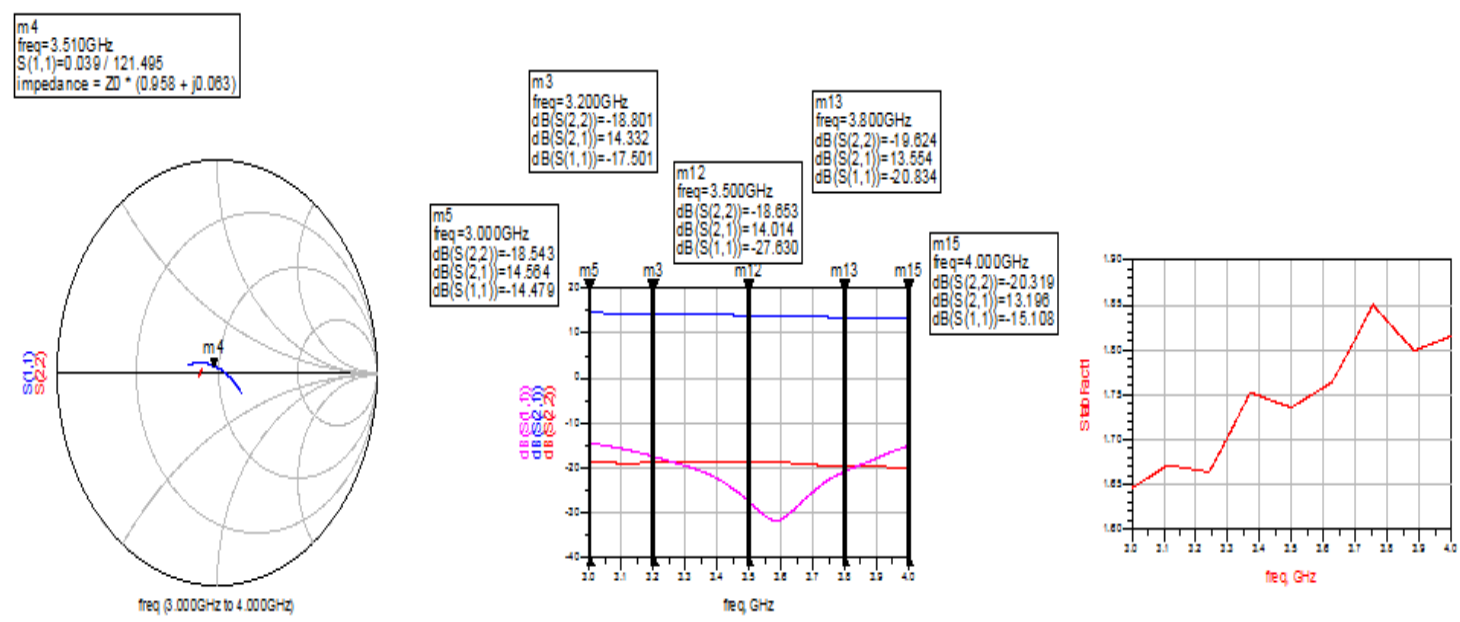

Fig14. Simulation results

\section{CONCLUSION:}

This paper presents the design of a Microwave Power amplifier with below mentioned specifications.Frequency range $=3 \mathrm{GHz}-4 \mathrm{GHz}$, Band width $=1 \mathrm{GHz}$, Gain $=$ Minimum $13 \mathrm{~dB}$, Flatness $= \pm 0.7 \mathrm{~dB}$, Input Return losses better than $-10 \mathrm{~dB}$, Output Return losses better than $30 \mathrm{Db}$, Power output $=30 \mathrm{dBm}, \mathrm{P} 1 \mathrm{~dB}=30 \mathrm{dBm}, \mathrm{PAE}=43 \%$.

The layout is generated from the designed circuit schematic using ADS, which is then printed on the RT duriod substrate using a Miller machine. All the components are assembled to realize the final circuit. Therefore the designing and fabrication of the MIC power amplifier is complete. 
International Journal on Cybernetics \& Informatics (IJCI) Vol. 5, No. 4, August 2016

\section{ACKNOWLEDGEMENTS}

The author would like to acknowledge SANGAM BALKE ,HOD of ATQC of GAETEC, Hyderabad for his constant mentoring during the complete course of the work.

\section{REFERENCES:}

[1] Guillermo Gonzalez, "Microwave Transistor Amplifiers, Analysis and Design", 2/e, Prentice Hall, 1996.

[2] David M Pozar, "Microwave Engineering", 4/e, Wiley India Pvt.Ltd, 2011.

[3] Ravender Goyal, "Monolithic Microwave Integrated Circuits", 1/e, Artech House Publishers, 1989.

[4] Vendelin, G.D., Design of Amplifiers and Oscillators by the S-Parameter Method, New York: J. Wiley and Sons, 1982.

[5] Samuel Y.Liao, "Microwave Devices and Circuits", 3/e, Pearson Education India, 1990. 